\title{
Borboletas frugívoras (Lepidoptera: Nymphalidae) no Horto Botânico Irmão Teodoro Luis, Capão do Leão, Rio Grande do Sul, Brasil
}

\author{
Janaína Madruga Silva ${ }^{1,3^{*}}$ \\ Samuel Kabke da Cunha ${ }^{2}$ \\ Eduardo José Ely e Silva ${ }^{3}$ \\ Flávio Roberto Mello Garcia ${ }^{1}$
${ }^{1}$ Laboratório de Ecologia de Insetos, Departamento de Zoologia e Genética Instituto de Biologia, Universidade Federal de Pelotas
${ }^{2}$ Laboratório de Parasitos e Vetores, Departamento de Microbiologia Instituto de Biologia, Universidade Federal de Pelotas
${ }^{3}$ Museu Entomológico Ceslau Biezanko, Departamento de Fitossanidade
Faculdade de Agronomia Eliseu Maciel, Universidade Federal de Pelotas
* Autor para correspondência
Rua Tiradentes, 1546, Centro, CEP 96010160, Pelotas - RS, Brasil
nina 0694@hotmail.com

Submetido em 22/05/2012

Aceito para publicação em 11/12/2012

\section{Resumo}

No Rio Grande do Sul, há carência de informações sistematizadas sobre a fauna de borboletas frugívoras. Este artigo teve por objetivo inventariar a guilda no Horto Botânico Irmão Teodoro Luis, ambiente caracterizado pela tensão ecológica entre a floresta estacional semidecidual e as formações pioneiras. As coletas foram realizadas em três áreas, cada uma contendo cinco armadilhas Van Someren-Rydon modificadas, iscadas com banana fermentada. As armadilhas permaneceram em campo durante seis dias consecutivos por mês, entre setembro de 2010 e agosto de 2011. Em 10.800 horas de esforço amostral, foram registrados 453 indivíduos distribuídos em 16 espécies, 15 gêneros e três subfamílias de Nymphalidae. Satyrinae foi a mais rica e abundante, sendo duas espécies deste grupo muito frequentes e dominantes. Os índices de riqueza indicaram entre $78 \%$ e $93 \%$ da fauna registrada, a curva de acúmulo de espécies condiz com esses valores e a diversidade foi de 2,02 (H'). Prepona pylene Hewitson, [1854] e Archaeoprepona demophoon (Hübner, [1814]) constituem novos registros para o Litoral Sul do estado.

Palavras-chave: Biodiversidade; Conservação; Frugivoria; Insetos; Preponini

\section{Abstract}

Frugivorous butterflies (Lepidoptera: Nymphalidae) in Irmao Teodoro Luis Botanical Garden, Capao do Leao, Rio Grande do Sul, Brazil. In Rio Grande do Sul, there's a lack of systematic information on frugivorous butterflies. This paper aimed survey the guild at Irmao Teodoro Luis Botanical Garden, an environment characterized by ecological tension between the semideciduous seasonal forest and the pioneer formations. The collections were made in three areas, each one containing five modified Van Someren-Rydon 
traps, baited with fermented banana. The traps remained on the field for six consecutive days per month, from September 2010 to August 2011. Within 10.800 hours of sampling effort, 453 individuals were recorded, distributed into 16 species, 15 genera, and 3 Nymphalidae subfamilies. Satyrinae was the richest and most abundant one, and two species from this group are very frequent and dominant. Richness rates indicated between $78 \%$ and $93 \%$ of the reported fauna, the species accumulation curve matches these values, and the diversity was $2.02\left(\mathrm{H}^{\prime}\right)$. Prepona pylene Hewitson, [1854] and Archaeoprepona demophoon (Hübner, [1814]) constitute new records for the Southern Coast of the state.

Key words: Biodiversity; Conservation; Frugivory; Insects; Preponini

\section{Introdução}

A ordem Lepidoptera possui aproximadamente 146.000 espécies descritas, 13\% correspondendo a borboletas (HEPPNER, 1991). No Brasil estão catalogadas 3.280 espécies de borboletas (DUARTE et al., 2012). Este grupo apresenta diversas relações ecológicas com outros organismos, cobre boa parte dos processos essenciais dos ecossistemas terrestres e contribui de forma considerável como biomassa alimentar para níveis tróficos superiores (FREITAS et al., 2003). Também estão entre os melhores grupos para monitoramento ambiental (DE VRIES et al., 1997; FREITAS et al., 2003).

As borboletas podem ser reunidas em guildas de acordo com o hábito alimentar dos indivíduos adultos. Borboletas frugívoras alimentam-se de frutas fermentadas, excrementos ou exsudados de plantas e animais em decomposição (DE VRIES, 1987; FREITAS et al., 2003). Neste conjunto incluem-se representantes de algumas subfamílias de Nymphalidae: Satyrinae (incluindo Brassolini e Morphini), Charaxinae, Biblidinae e Nymphalinae (DE VRIES, 1987; FREITAS et al., 2003).

Nymphalidae é composta por borboletas muito admiradas e bem estudadas, possui ampla distribuição mundial e talvez seja a família de borboletas mais diversificada em termos de hábito e morfologia, apresentando o maior número de espécies conhecidas (DUARTE et al., 2012). Segundo os mesmos autores, existem cerca de 800 ninfalídeos no Brasil. Aproximadamente 335 são atraídos por iscas e destes 11 estão ameaçados (LEWINSOHN et al., 2005).

Diversos estudos relacionam integrantes de Nymphalidae ao Rio Grande do Sul (MARCHIORI;
ROMANOWSKI, 2006a; 2006b; DESSUY; MORAIS, 2007; ISERHARD et al., 2010; ROSA et al., 2011). No Litoral Sul, estes lepidópteros foram registrados por Biezanko e Freitas (1938), Biezanko (1949; 1958; 1959; 1960a; 1960b) e Krüger e Silva (2003). Porém, apenas alguns trabalhos utilizam metodologia específica para frugívoros (TESTON; CORSEUIL, 2002; QUADROS et al., 2004; ROMAN et al., 2010; PEDROTTI et al., 2011; SANTOS et al., 2011)

No Litoral Sul do Rio Grande do Sul, não há registros de estudos utilizando armadilhas atrativas para a captura de borboletas frugívoras. Tendo em vista a importância do grupo e esta lacuna em seu conhecimento, este trabalho teve como objetivo inventariar a guilda de borboletas frugívoras no Horto Botânico Irmão Teodoro Luis.

\section{Material e Métodos}

O estudo foi realizado no Horto Botânico Irmão Teodoro Luis ( $\left.31^{\circ} 48^{\prime} 58^{\prime \prime} \mathrm{S}, 52^{\circ} 25^{\prime} 55^{\prime \prime} \mathrm{W}\right)$, unidade de preservação federal permanente de responsabilidade da Universidade Federal de Pelotas, regulamentada pela Portaria Ministerial 77 no ano de 1964. Possui 23 ha de mata nativa circundada por áreas de Formações Pioneiras (banhados) e por Estepe Gramíneo-Lenhosa (campos). O local apresenta um longo histórico de interferência antrópica, tendo passado por um projeto paisagístico na primeira metade do século XX (LUIS; BERTELS, 1951). Somente há poucas décadas a área tem sido usada apenas para trabalhos acadêmicos e a mata tem se regenerado.

O Horto situa-se no município do Capão do Leão, Estado do Rio Grande do Sul, região geomorfológica da Planície Costeira, Litoral Sul (Figura 1). Pertence ao Bioma Campos Sulinos e se encontra na fisionomia 
das Formações Pioneiras com forte influência da Floresta Estacional Semi-Decidual (VELOSO et al., 1991). O clima é Cfa (mesotérmico, sempre úmido, com verões quentes) de acordo com a classificação de Köppen (MORENO, 1961). Segundo a Estação Agroclimatológica de Pelotas (EMBRAPA, 2010), as médias normais de temperatura e precipitação pluviométrica são de $23^{\circ} \mathrm{C}$ e $125 \mathrm{~mm}$ no verão, $18^{\circ} \mathrm{C}$ e $100 \mathrm{~mm}$ no outono, $13^{\circ} \mathrm{C}$ e $123 \mathrm{~mm}$ no inverno e $17^{\circ} \mathrm{C}$ e $108 \mathrm{~mm}$ na primavera.

Foram escolhidas três áreas amostrais (I, II e III) com distância de no mínimo $200 \mathrm{~m}$ uma da outra a $30 \mathrm{~m}$ da beira da mata. Em cada área, foram dispostas cinco armadilhas portáteis do tipo Van Someren - Rydon modificadas (Figura 2) de acordo com Uehara-Prado et al. (2007), colocadas na vegetação arbustiva entre 1,50 a 2,0 m de altura e distantes umas das outras em no mínimo $15 \mathrm{~m}$. As armadilhas permaneceram em campo durante seis dias consecutivos por mês, com verificações realizadas a cada $48 \mathrm{~h}$ para a manutenção de isca e captura de indivíduos, entre setembro de 2010 e agosto de 2011. Para a atração das borboletas foi utilizado um preparado de banana, açúcar mascavo e água, fermentado por $48 \mathrm{~h}$.

FIGURA 1: Localização do Horto Botânico Irmão Teodoro Luis ( $31^{\circ} 48^{\prime} 58^{\prime}$ 'S, 52 $\left.25^{\prime} 55^{\prime \prime} \mathrm{W}\right)$, Capão do Leão, Rio Grande do Sul, Brasil. Fonte: ABREU, 2006.

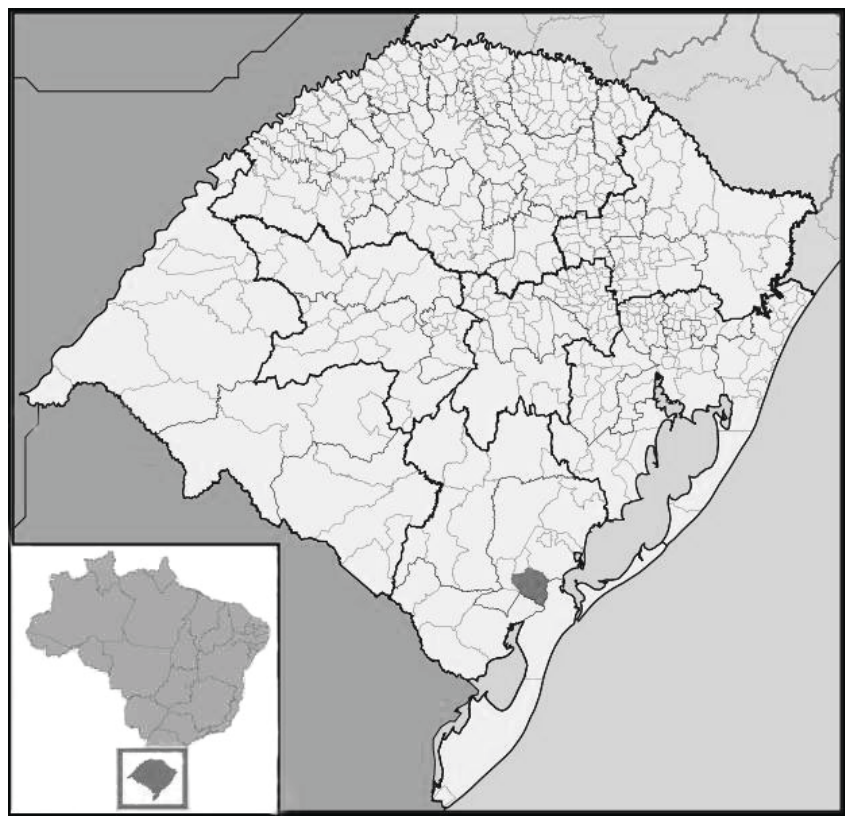

Espécimes de fácil identificação no campo foram marcados numericamente, fotografados e liberados. Indivíduos de identificação incerta e exemplares testemunhos foram mortos em câmaras mortíferas com acetato de etila. Após, foram acondicionados em envelopes entomológicos e encaminhados ao Museu Entomológico Ceslau Biezanko, onde foram preparados a seco, identificados e depositados.

O esforço amostral foi calculado multiplicando-se o número de armadilhas pelas 10h/dia de amostragem (tempo que se supõem as borboletas estarem ativas). A identificação dos espécimes foi realizada utilizando-se bibliografia especializada (D'ABRERA, 1988; 1994; 1995; BROWN JR., 1992; CANALS, 2000; 2003) e comparação com exemplares da Coleção do Museu Entomológico Ceslau Biezanko. A nomenclatura foi atualizada segundo Lamas (2004) e a sistemática conforme Wahlberg et al. (2009).

FIGURA 2: Armadilha portátil do tipo Van Someren - Rydon modificada de acordo com Uehara-Prado et al. (2007).

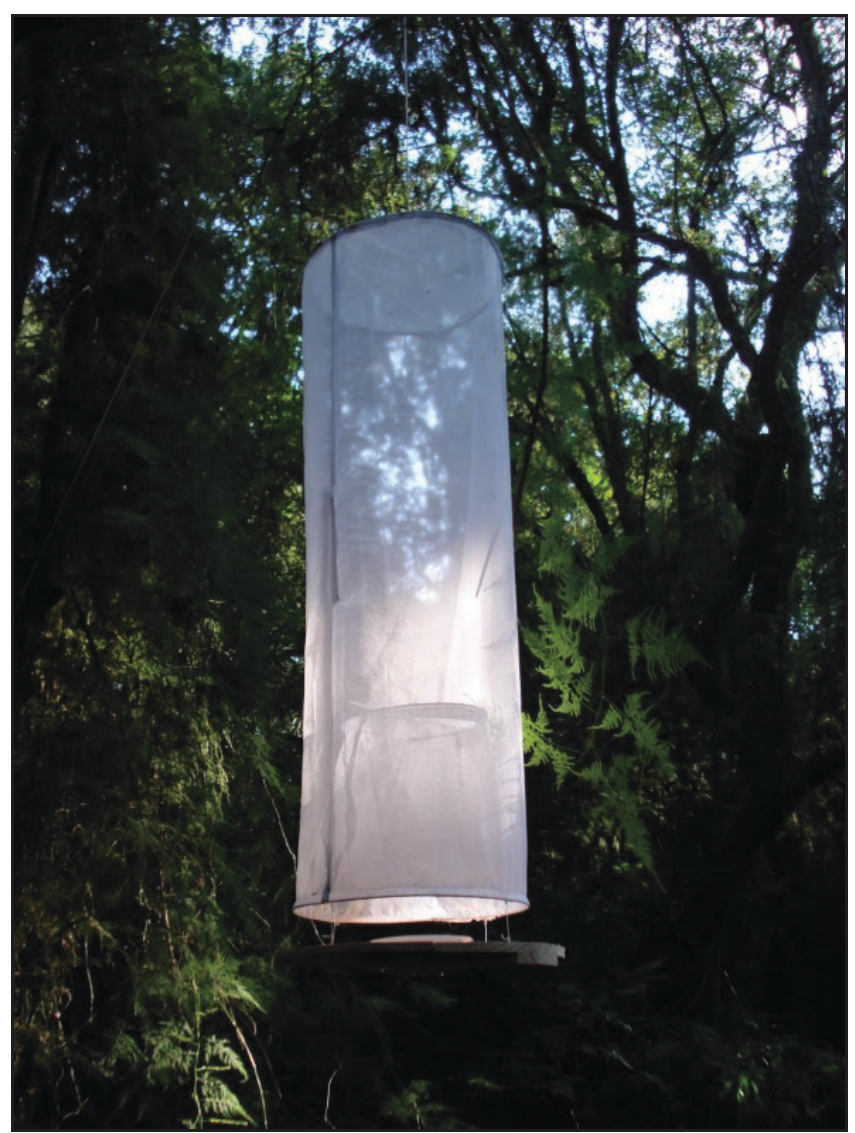


As análises foram realizadas considerando-se a abundância específica $(\mathrm{N})$ e a riqueza $(\mathrm{S})$, sendo construída uma curva de acúmulo de espécies. A frequência relativa $(\mathrm{Fr})$ foi obtida pela razão entre o número de indivíduos da espécie estudada e o número total de indivíduos da amostra multiplicado por 100 . Foi calculado um intervalo de confiança (5\%), e as espécies foram classificadas em pouco frequente ( $\mathrm{Fr}$ $<\mathrm{LI}$ ), frequente ( $\mathrm{LI}<\mathrm{Fr}<\mathrm{LS}$ ) e muito frequente (Fr $>$ LS) (SILVEIRA NETO et al., 1976). Foram consideradas singletons e doubletons as espécies que apresentaram um ou dois indivíduos na amostra total respectivamente (SANTOS, 2003). O limite de dominância foi determinado multiplicando o inverso da riqueza por 100 (SAKAGAMI; LAROCA, 1971). Foram consideradas dominantes as espécies em que a frequência ultrapassa o limite calculado.

Para calcular a diversidade foi utilizado o índice de Shannon-Wiener (H'). A riqueza e seus intervalos de confiança $(95 \%)$ foram obtidos pelos estimadores analíticos Chao 1, Chao 2, Jackknife 1 (Jack 1), Jackknife 2 (Jack 2) e Bootstrap. Para os cálculos, construção de gráficos e tabelas foram utilizados os programas Excel e EstimateS 8.0 (COLWELL, 2006).

\section{Resultados e Discussão}

Em um total de $10.800 \mathrm{~h}$ de esforço amostral foram registrados 453 indivíduos de 16 espécies, distribuídos em 15 gêneros e três subfamílias (Biblidinae, Charaxinae e Satyrinae) de Nymphalidae. Três espécies foram classificadas como singleton e uma como doubleton (Tabela 1). Também foram coletados dois indivíduos de Doxocopa laurentia (Godart, [1824]) (Apaturinae), porém, por não serem frugívoros estritos não foram considerados neste estudo.

Do total de indivíduos amostrados, 441 (97,35\%) pertencem à subfamília Satyrinae, $11(2,43 \%)$ à Charaxinae e um $(0,22 \%)$ à Biblidinae. Além de mais abundante, Satyrinae também demonstrou ser a subfamília mais rica $(S=11)$, seguida de Charaxinae ( $S=4)$ e Biblidinae $(S=1)$. Modelo de representatividade semelhante foi verificado por Pedrotti et al. (2011) na

TABELA 1: Abundância (N) de borboletas frugívoras capturadas com armadilhas Van Someren - Rydon modificadas, no Horto Botânico Irmão Teodoro Luis entre os meses de setembro de 2010 a agosto de 2011.

\begin{tabular}{clr}
\hline & \multicolumn{1}{c}{ Taxa } & N \\
\hline Nymphalidae & & 1 \\
Biblidinae & & \\
Biblidini & Hamadryas februa (Hübner, [1823]) & 2 \\
Charaxinae & & 1 \\
Preponini & Archaeoprepona demophoon (Hübner, [1814]) & 3 \\
& Prepona pylene Hewitson, [1854] & 5 \\
Anaeini & Memphis moruus stheno (Prittwitz, 1865) & 1 \\
& Zaretis itys (Cramer, 1777) & 114 \\
Satyrinae & Blepolenis batea (Hübner, [1821]) & 14 \\
Brassolini & Caligo martia (Godart, [1824]) & 17 \\
& Catoblepia amphirhoe (Hübner, [1825]) & 28 \\
& Eryphanis reevesii (Doubleday, [1849]) & 23 \\
& Opsiphanes invirae (Hübner, [1808]) & 57 \\
Morphini & Morpho epistrophus (Fabricius, 1796) & 4 \\
Satyrini & Capronnieria galesus (Godat, [1824]) & 64 \\
& Hermeuptychia hermes (Fabricius, 1775) & 115 \\
& Paryphthimoides eous (Butler, 1867) & 4 \\
\hline & Paryphthimoides poltys (Prittwitz, 1865) & $\mathbf{4 5 3}$ \\
\hline Total & Yphthimoides celmis (Godart, [1824]) & \\
\hline
\end{tabular}


Floresta Ombrófila Mista do Rio Grande do Sul. Este padrão pode estar relacionado ao fato deste levantamento seguir a mesma sistemática do presente estudo, considerando Brassolini e Morphini tribos de Satyrinae.

Satyrinae é uma subfamília de ampla diversidade, biologia e distribuição, compõem um terço de todas as espécies da família Nymphalidae (PEÑA; WAHLBERG, 2008). A região neotropical é responsável pela maior riqueza de satiríneos do mundo (D’ABRERA, 1988). Suas principais plantas hospedeiras são monocotiledôneas (DE VRIES, 1987; PEÑA; WAHLBERG, 2008), abundantes nas clareiras, mas principalmente na borda e nos arredores da mata do Horto. Por ser um fragmento relativamente grande, porém cortado por trilhas e clareiras, torna-se propicio para o desenvolvimento e manutenção deste grupo, gerando recursos tanto para juvenis quanto para adultos.

Quatro espécies $(25,0 \%)$ foram consideradas muito frequentes, duas $(12,50 \%)$ frequentes e $10(62,50 \%)$ pouco freqüentes (Figura 3). Das dez espécies mais abundantes citadas para o Rio Grande do Sul (MORAIS et al., 2007), apenas Hermeuptychia hermes (Fabricius,
1775) foi registrada neste levantamento, estando classificada como pouco frequente. Isto se deve ao fato de que dentre estas dez espécies, apenas três são frugívoras, portanto passíveis de serem coletadas pelo método utilizado neste trabalho. $H$. hermes ocorre no extrato herbáceo de florestas com clareiras (CANALS, 2000). Provavelmente, a localização das armadilhas (interior de mata) tenha influenciado no baixo número de exemplares coletados.

As espécies Paryphthimoides poltys (Prittwitz, 1865) e Caligo martia (Godart, [1824]) foram as únicas dominantes. No trabalho de Krüger e Silva (2003), realizado em Pelotas, Capão do Leão e Morro Redondo, foram apontadas apenas oito espécimes de $P$. poltys e nove de $C$. martia. Neste estudo foram registradas 45 espécies de borboletas frugívoras, destas, 14 espécies encontradas no Horto. Este resultado está associado principalmente às metodologias empregadas por estes autores, coletas com rede entomológica e visita a coleções, bem como a diferença no tamanho da área abrangida e no tempo de amostragem. Estas informações demonstram a importância das armadilhas atrativas para levantamentos de borboletas frugívoras e sua

FIGURA 3: Frequência relativa (\%) das borboletas frugívoras capturadas com armadilhas Van Someren - Rydon modificadas, no Horto Botânico Irmão Teodoro Luis entre os meses de setembro de 2010 a agosto de 2011.

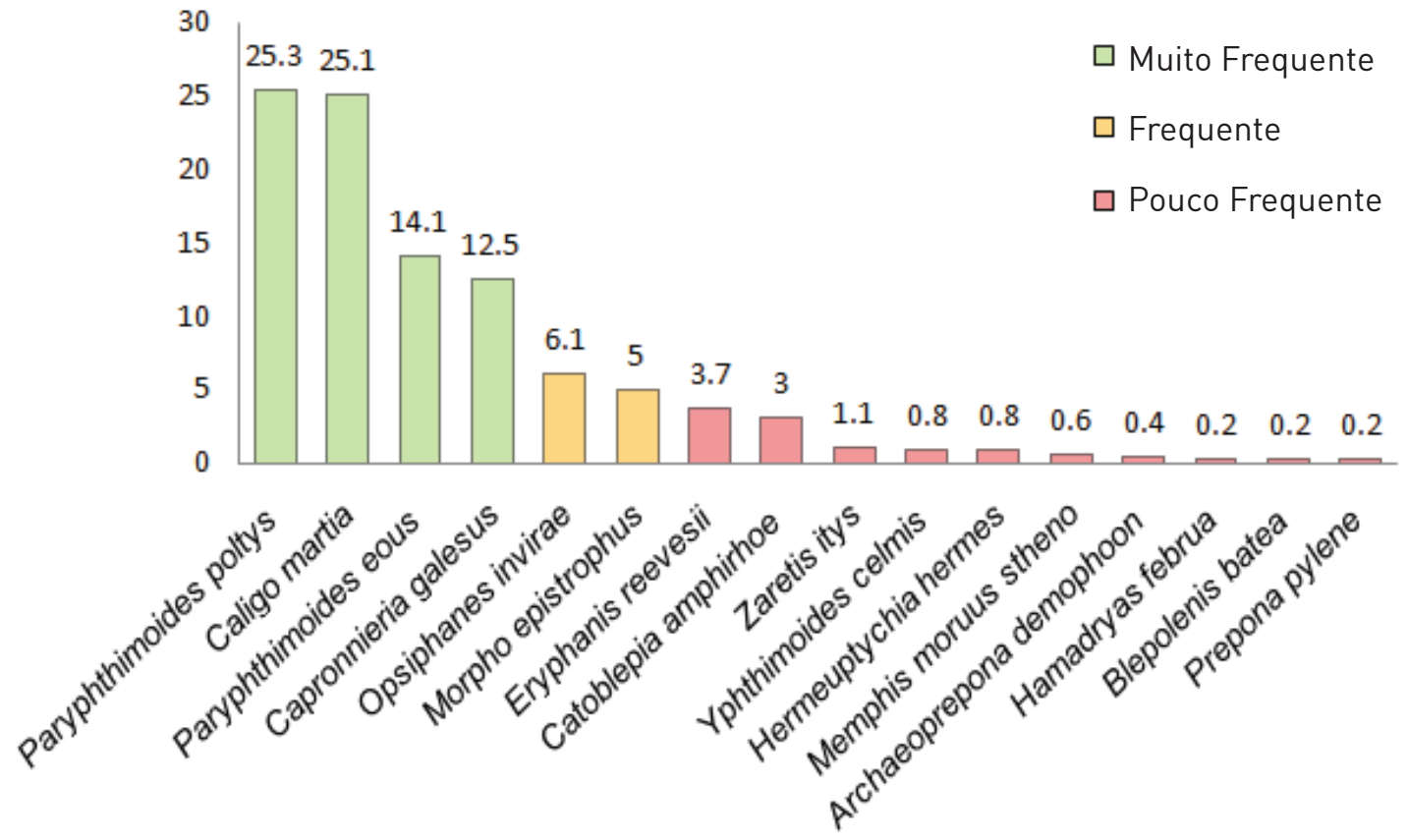


FIGURA 4: Número acumulado de espécies de borboletas frugívoras amostradas no Horto Botânico Irmão Teodoro Luis entre os meses de setembro de 2010 a agosto de 2011.

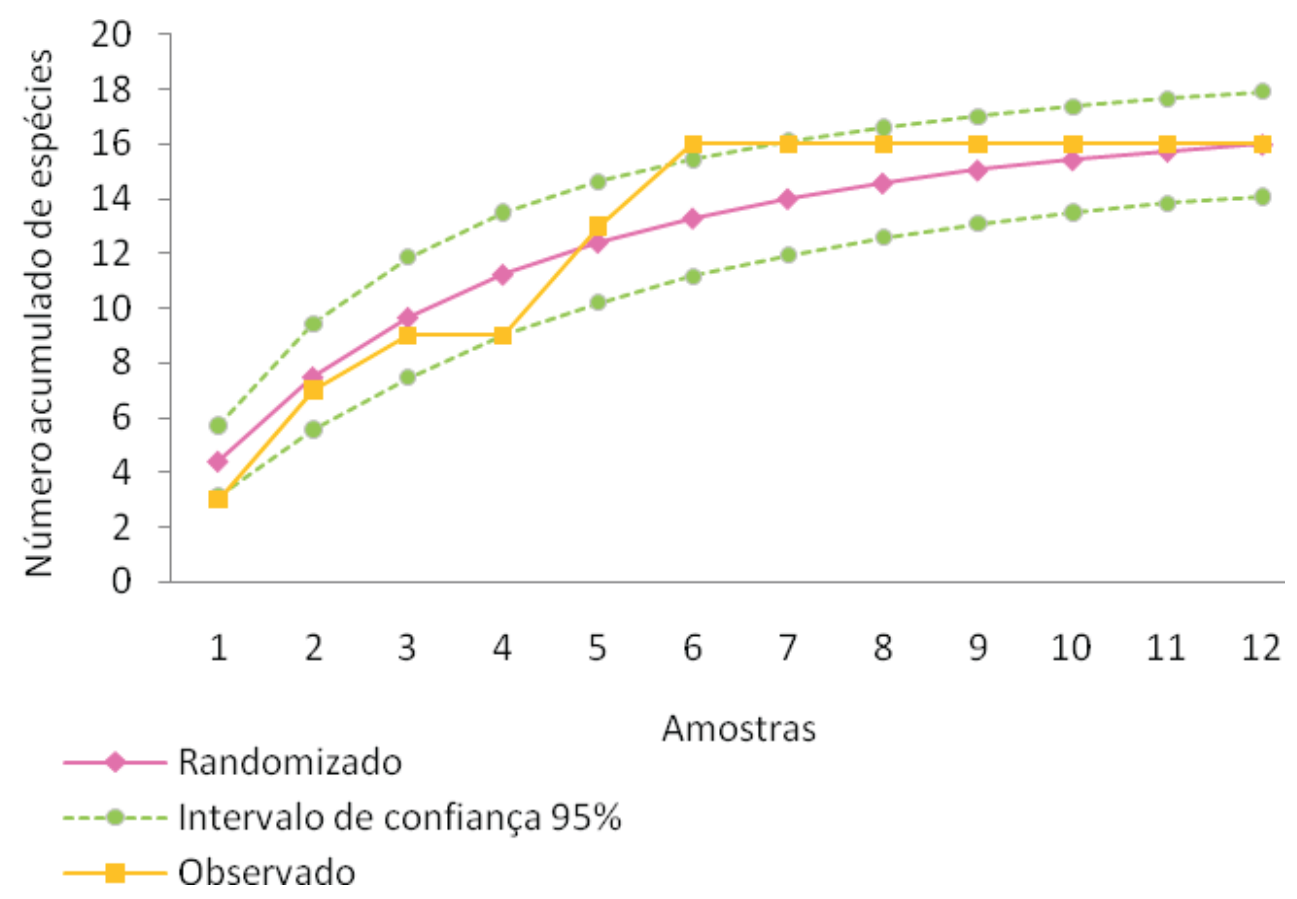

necessidade em associação a outras técnicas para um levantamento faunístico mais preciso.

Obteve-se o valor de 2,02 pelo índice de diversidade de Shannon-Wiener (H'). Para a riqueza, o estimador Chao 1 apresentou o maior resultado $(\mathrm{S}=21)$, indicando que $78 \%$ da fauna foi amostrada e o estimador Chao 2 o menor ( $\mathrm{S}=17$ ), apontando $93 \%$ da fauna amostrada. Os demais estimadores obtiveram valores intermediários: Jack $1(S=19)$, Bootstrap $(S=18)$ e Jack $2(S=18)$. A curva de acúmulo de espécies obtida condiz com os valores encontrados através dos estimadores de riqueza, demonstrando que poucas espécies ainda estão por ser amostradas (Figura 4).

Ainda para a riqueza, o valor alcançado foi relativamente baixo em comparação a outros trabalhos realizados somente com armadilhas no Rio Grande do Sul (ROMAN et al., 2010; PEDROTTI et al., 2011). Sendo muito inferior aos valores encontrados em estudos que juntamente com esta metodologia utilizaram rede entomológica (TESTON; CORSEUIL, 2002; QUADROS et al., 2004; SANTOS et al., 2011). No entanto, conclusões podem ser precipitadas, visto que os trabalhos analisados são de diferentes ambientes e não seguem uma metodologia padronizada.

Das espécies coletadas, duas foram novos registros para o Litoral Sul do Estado: Prepona pylene Hewitson, [1854] e Archaeoprepona demophoon (Hübner, [1814]) (Charaxinae) (Figura 5). Nas listas recentes, estas espécies são citadas para o Nordeste do Rio Grande do Sul (PEDROTTI et al., 2011; SANTOS et al., 2011). Esta subfamília é composta por borboletas de corpos robustos e vôos rápidos, características de dossel de florestas (FLECK et al., 2007), particularidade que dificulta sua coleta através de rede entomológica. Este pode ser o motivo pelo qual os estudos que mencionam A. demophoon e P. pylene tenham utilizado armadilhas atrativas.

Dos 453 espécimes registrados, 192 indivíduos de nove espécies foram capturados, marcados e soltos, sendo 57 o número de recapturas (Tabela 2). Segundo Uehara-Prado et al. (2005), o baixo número de recapturas pode estar ligado a características particulares de cada espécie, como tamanho populacional, longevidade, capacidade de vôo e 
FIGURA 5: Novos registros de borboletas frugívoras para o Litoral Sul do Rio Grande do Sul, capturadas com armadilhas Van Someren - Rydon modificadas, no Horto Botânico Irmão Teodoro Luis entre os meses de setembro de 2010 a agosto de 2011.
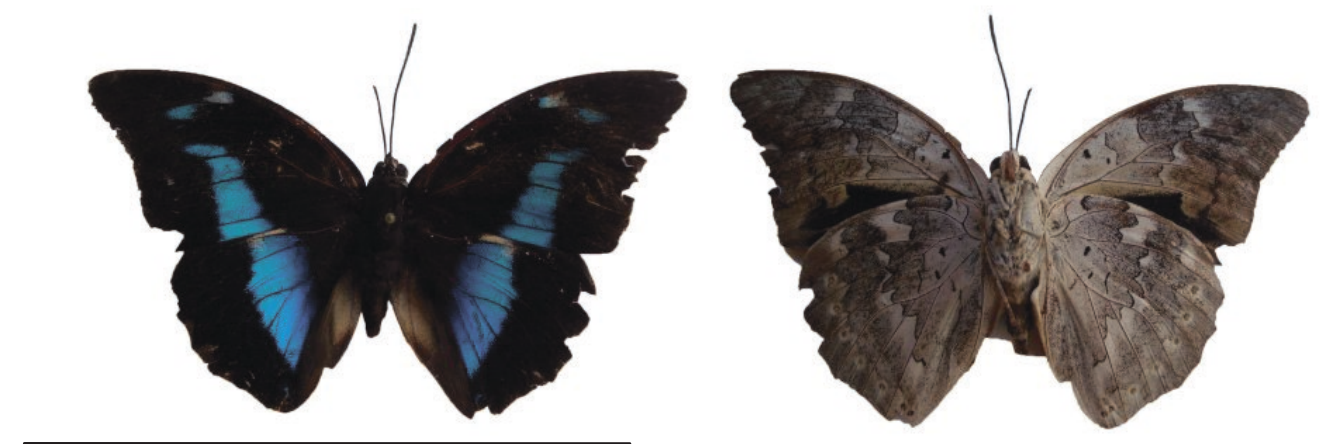

$9,2 \mathrm{~cm}$

Archaeoprepona demophoon (Hübner, [1814])
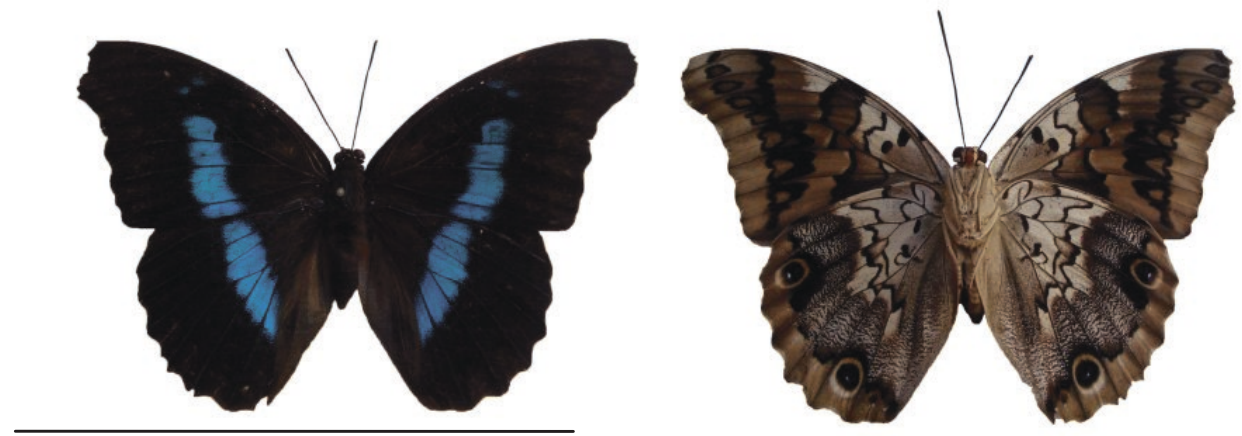

$10,3 \mathrm{~cm}$

Prepona pylene Hewitson, [1854]

TABELA 2: Captura, marcação e recaptura de borboletas frugívoras utilizando armadilhas Van Someren - Rydon modificadas, no Horto Botânico Irmão Teodoro Luis entre os meses de setembro de 2010 a agosto de 2011.

\begin{tabular}{lccc}
\hline \multicolumn{1}{c}{ Espécie } & Captura & Marcação & Recaptura \\
\hline Caligo martia (Godart, [1824]) & 114 & 101 & $46 \%$ \\
Capronnieria galesus (Godat, [1824]) & 57 & 21 & - \\
Opsiphanes invirae (Hübner, [1808]) & 28 & 25 & $16 \%$ \\
Morpho epistrophus (Fabricius, 1796) & 23 & 14 & $14 \%$ \\
Eryphanis reevesii (Doubleday, [1849]) & 17 & 14 & $14 \%$ \\
Catoblepia amphirhoe (Hübner, [1825]) & 14 & 12 & $25 \%$ \\
Zaretis itys (Cramer, 1777) & 5 & 3 & - \\
Memphis moruus stheno (Prittwitz, 1865) & 3 & 1 & - \\
Archaeoprepona demophoon (Hübner, [1814]) & 2 & 1 & - \\
\hline Total & $\mathbf{2 6 3}$ & $\mathbf{1 9 2}$ & $\mathbf{5 7}$ \\
\hline
\end{tabular}


territorialidade, além de traumas decorrentes da captura e do manuseio. Com relação aos prováveis danos físicos e estresse causados pela técnica, para o objetivo proposto as vantagens mostram-se superiores. Por mais que muitos indivíduos possam ter se tornados vulneráveis ou até mesmo relutantes após sua soltura, menos foram retirados da população com relação ao que ocorreria em um trabalho convencional.

Ademais, a técnica demonstrou ser uma aliada em estudos que visam obter informações como de longevidade e deslocamento de espécies. Caligo martia obteve o maior número de recapturas, o que pode estar relacionado à sua grande abundancia e ao número de exemplares marcados. Entre as espécies analisadas, sete espécimes de $C$. martia exibiram intervalo de um mês entre uma captura e outra, sugerindo seu tempo de vida. Três indivíduos desta espécie foram recapturados em uma área diferente da que foram marcados. Além de C. martia, Opsiphanes invirae apresentou um indivíduo com deslocamento de área.

Não foram recapturados indivíduos de quatro espécies: Zaretis itys, Memphis moruus stheno, Archaeoprepona demophoon e Capronnieria galesus. As três primeiras tiveram um ou poucos exemplares marcados, o que pode justificar a ausência de novas capturas. Porém, C. galesus obteve 21 marcações. Esta espécie diverge das demais pelo seu tamanho reduzido e pela sua aparente fragilidade, podendo ter sido prejudicada durante seu manuseio.

\section{Agradecimentos}

Meu sincero agradecimento a todos os integrantes e colaboradores que contribuíram para que este trabalho fosse possível. Em especial aos meus amores que participaram através de incentivo, apoio e auxílio nas diversas etapas do seu desenvolvimento. Agradeço também aos revisores anônimos pelas valiosas sugestões.

\section{Referências}

ABREU, R. L. de. Rio Grande do Sul. 2006. Disponível em: $\quad<$ http://pt.wikipedia.org/wiki/Ficheiro:RioGrandedoSul MesoMicroMunicip.svg >. Acesso em: 18 jun. 2010.
BIEZANKO, C. M. Acraeidae, Heliconidae e Nymphalidae de Pelotas e seus arredores. Arquivos de Entomologia Série A, Pelotas, p. 1-16, 1949.

BIEZANKO, C. M. Pieridae da Zona Sueste do Rio Grande do Sul. Arquivos de Entomologia Série A, Pelotas, p. 1-15, 1958.

BIEZANKO, C. M. Papilionidae da Zona Sueste do Rio Grande do Sul. Arquivos de Entomologia Série A, Pelotas, p. 1-17, 1959

BIEZANKO, C. M. Danaidae et Ithomiidae da Zona Sueste do Rio Grande do Sul. Arquivos de Entomologia Série A, Pelotas, p. 1-6, 1960a.

BIEZANKO, C. M. Satyridae, Morphidae et Brassolidae da Zona Sueste do Rio Grande do Sul. Arquivos de Entomologia Série A, Pelotas, p. 1-13, 1960 b.

BIEZANKO, C. M.; FREITAS, R. G. Catálogo dos insetos encontrados na cidade de Pelotas e seus arredores. Fasc. 1 Lepidópteros. Boletim da Escola de Agronomia Eliseu Maciel, Pelotas, n. 25, p. 1-32, 1938.

BROWN JR., K. S. Borboletas da Serra do Japi: diversidade, hábitats, recursos alimentares e variação temporal. In: MORELLATO, L. P. C. (Org.). História Natural da Serra do Japi - Ecologia e preservação de uma área florestal no sudeste do Brasil. São Paulo: Editora da Universidade de Campinas, 1992. p. 142-186.

CANALS, G. Mariposas Bonaerenses. Buenos Aires: L.O.L.A., 2000. 347 p.

CANALS, G. Mariposas de Misiones. Buenos Aires: L.O.L.A., 2003. $492 \mathrm{p}$.

COLWELL, R. K. Estimates 8.0 estatistical estimation of species richness and shared species from samples. 2006. Disponível em: $<$ http://viceroy.eeb.ucon.edu/estimates $>$. Acesso em: 10 out. 2011.

D'ABRERA, B. Butterflies of the Neotropical Region. Part V. Nymphalidae \& Satyridae. Victoria: Hill House, 1988. 877 p.

D'ABRERA, B. Butterflies of the Neotropical region. Part VI. Riodinidae. Victoria: Hill House, 1994. 10967 p.

D'ABRERA, B. Butterflies of the Neotropical Region. Part VII. Lycaenidae. Victoria: Hill House, 1995. 1270 p.

DE VRIES, P. J. The butterflies of Costa Rica and their natural history: Papilionidae, Pieridae and Nymphalidae. New Jersey: Princeton University, 1987. 327 p.

DE VRIES, P. J.; MURRAY, D.; LANDE, R. Species diversity in vertical, horizontal, and temporal dimensions of a fruit-feeding butterfly community in an Ecuadorian rainforest. Biological Journal of the Linnean Society, London, v. 62, n. 3, p. 343-364, 1997.

DESSUY, M. B.; MORAIS, A. B. B. Diversidade de borboletas (Lepidoptera, Papilionoidea e Hesperioidea) em fragmentos de Floresta Estacional Decidual em Santa Maria, Rio Grande do Sul, Brasil. Revista Brasileira de Zoologia, Curitiba, v. 24, n. 1, p. 108120, 2007

DUARTE, M.; MARCONATO, G.; SPECHT, A.; CASAGRANDE, M. M. Lepidoptera. In: RAFAEL, J. A.; MELO, G. A. R.; CARVALHO, C. J. B. de; CASARI, S. A.; CONSTANTINO, R. (Ed.). Insetos do Brasil: diversidade e taxonomia. Ribeirão Preto: Holos, 2012. p. 625-682.

EMBRAPA - Laboratório de Agrometeorologia. Estação Agroclimatológica de Pelotas. 2010. Disponível em: $<$ http://www. 
cpact.embrapa.br/agromet/estacao/mensal.html>. Acesso em: 18 jun. 2010.

FLECK, T.; BECKER, R. G.; MACHADO, I. F.; SILVA, R. B. da; BARBIERI, R. L. (Ed.). Borboletas da SIX: guia de borboletas frugívoras. Pelotas: Embrapa Clima Temperado, 2007. 108 p.

FREITAS, A. V. L.; FRANCINI, R. B.; BROWN JR., K. S. Insetos como indicadores ambientais. In: CULLEN, JR.; RUDRAN, R.; VALLADARES-PADUA, C. (Ed.). Métodos de estudos em biologia da conservação e manejo da vida silvestre. Curitiba: Editora UFPR, 2003. p. 125-152.

HEPPNER, J. B. Faunal regions and the diversity of Lepidoptera. Tropical Lepidoptera, Gainesville, v. 2, p. 1-85, 1991.

ISERHARD, C. A.; QUADROS, M. T.; ROMANOWSKI, H. P.; MEDONÇA JR., M. S. Borboletas (Lepidoptera: Papilionoidea e Hesperioidea) ocorrentes em diferentes ambientes na Floresta Ombrófila Mista e nos Campus de Cima da Serra do Rio Grande do Sul, Brasil. Biota Neotropica, Campinas, v. 10, n. 1, p. 309-320, 2010.

KRÜGER, C. P.; SILVA, E. J. E. Papilionoidea (Lepidoptera) de Pelotas e seus arredores, Rio Grande do Sul, Brasil. Entomología y Vectores, Rio de Janeiro, v. 10, n. 1, p. 31-45, 2003.

LAMAS, G. Checklist: Part 4A. Hesperioidea - Papilionoidea. In: HEPPNER, J. B. (Ed.). Atlas of Neotropical Lepidoptera. Gainesville: Scientific Publishers, 2004. 439 p.

LEWINSOHN, M. T.; FREITAS, A. V. L.; PRADO, P. I. Conservação de invertebrados terrestres e seus habitats no Brasil. Megadiversidade, Belo Horizonte, v. 1, n. 1, p. 62-69, 2005.

LUIS, T.; BERTELS, A. Horto Botânico do Instituto Agronômico do Sul (Pelotas). Pelotas: Instituto Agronômico do Sul, 1951. 98 p. MARCHIORI, M. O.; ROMANOWSKI, H. P. Borboletas (Lepidoptera, Papilionoidea e Hesperioidea) do Parque Estadual do Espinilho e entorno, Rio Grande do Sul, Brasil. Revista Brasileira de Zoologia, Curitiba, v. 23, n. 4, p. 1029-1037, 2006 a.

MARCHIORI, M. O.; ROMANOWSKI, H. P. Species composition and diel variation of a butterfly taxocene (Lepidoptera, Papilionoidea and Hesperioidea) in a restinga forest at Itapuã State Park, Rio Grande do Sul, Brazil. Revista Brasileira de Zoologia, Curitiba, v. 23, n. 2, p. 443-454, 2006 b.

MORAIS, A. B. B.; ROMANOWSKI, H. P.; ISERHARD, C. A.; MARCHIORI, M. O.; SEGUI, R. Mariposas del Sur de Sudamérica (Lepidoptera: Papilionoidea e Hesperioidea). Ciência \& Ambiente, Santa Maria, v. 35, p. 29-46, 2007.

MOREnO, J. A. Clima do Rio Grande do Sul. Porto Alegre: Secretaria da Agricultura, 1961. $41 \mathrm{p}$.

PEDROTTI, V. S.; BARROS, M. P.; ROMANOWSKI, H P.; ISERHARD, C. A. Borboletas frugívoras (Lepidoptera, Nymphalidae) ocorrentes em um fragmento de Floresta Ombrófila Mista no Rio Grande do Sul, Brasil. Biota Neotropica, Campinas, v. 11, n. 1, p. 385-390, 2011.

PEÑA, C.; WAHLBERG, N. Prehistorical climate change increased diversification of a group of butterflies. Biology Letters, London, v. 4, p. $274-278,2008$.
QUADROS, F. C.; DORNELES, A. L.; CORSEUIL, E. Ninfalídeos (Lepidoptera) ocorrentes no norte da planície costeira do Rio Grande do Sul, Brasil. Biociências, Porto Alegre, v. 12, n. 2, p. 147-167, 2004

ROMAN, M.; GARLET, J.; COSTA, E. C. Levantamento populacional e comportamento de vôo de borboletas (Lepidoptera) em um remanescente florestal em São Sepé, RS. Ciência Florestal, Santa Maria, v. 20, n. 2, p. 283-294, 2010.

ROSA, P. L. P.; CHIVA, E. Q.; ISERHARD, C. A. Borboletas (Lepidoptera: Papilionoidea e Hesperioidea) do Sudoeste do Pampa Brasileiro, Uruguaiana, Rio Grande do Sul, Brasil. Biota Neotropica, Campinas, v. 11, n. 1, p. 355-360, 2011.

SAKAGAMI, S. F.; S. LAROCA. Relative abundance, phenology and flower visits of apid bees in eastern Paraná, Southern Brazil (Hymenoptera, Apidae). Kontyû, Tokyo, v. 39, n. 3, p. 217-230, 1971.

SANTOS, A. J. Estimativa de riqueza em espécies. In: CULLEN, Jr.; RUDRAN, R.; VALLADARES-PADUA, C. (Orgs). Métodos de estudos em biologia da conservação e manejo da vida Silvestre. Curitiba: Editora da Universidade Federal do Paraná, 2003. p. 19-41.

SANTOS, J. P.; ISERHARD, C. A.; TEIXEIRA, M. O.; ROMANOWSKI, H. P. Fruit-feeding butterflies guide of subtropical Atlantic Forest and Araucaria Moist Forest in State of Rio Grande do Sul, Brazil. Biota Neotropica, Campinas, v. 11, n. 3, p. 253-274, 2011.

SILVEIRA NETO, S.; NAKANO, O.; BARBIN, D.; VILLA NOVA, N. A. Manual de ecologia dos insetos. Piracicaba: Ceres, 1976. 419 p.

TESTON, J. A.; CORSEUIL, E. Borboletas (Lepidoptera, Rhopalocera) ocorrentes no Centro de Pesquisa e Conservação da Natureza Pró-Mata. 3: Nymphalidae. Divulgações do Museu de Ciências e Tecnologia UBEA / PUCRS, Porto Alegre, n. 7, p. 1-208, 2002.

UEHARA-PRADO, M.; BROWN JR., K. S.; LUCCI FREITAS, A. V. Biological traits of frugivorous butterflies in a fragmented and a continuous landscape in the south Brasilian Atlantic Forest. Journal of the Lepidopterists' Society, New Haven, v. 59, n. 2, p. 96-106, 2005.

UEHARA-PRADO, M.; BROWN JR., K. S.; LUCCI FREITAS, A. V. Species richness, composition and abundance of fruit-feeding butterflies in the Brazilian Atlantic Forest: comparison between a fragmented and a continuous landscape. Global Ecology and Biogeography, Malden, n. 16, p. 43-54, 2007.

VELOSO, H. P.; RANGEL FILHO, A. L. R.; LIMA, J. C. A. Classificação da vegetação brasileira, adaptada a um sistema universal. Rio de Janeiro: IBGE, 1991. 124 p.

WAHLBERG, N.; LENEVEU, J.; KODANDARAMAIAH, U.; PEÑA, C.; NYLIN, S.; FREITAS, A. V. L.; BROWER, A. V. Z. Nymphalid butterflies diversify following near demise at the Cretaceous/Tertiary boundary. Proceedings of the Royal Society B, London v. 276, p. 4295-4302, 2009. 